Erschienen in: Engelberg, Stefan/Holler, Anke/Proost, Kristel (Hrsg.):

Sprachliches Wissen zwischen Lexikon und Grammatik. - Berlin, Boston: de

Gruyter, 2011. S. 181-210. (Institut für Deutsche Sprache. Jahrbuch 2010), https://doi.org/10.1515/9783110262339.181

Anatol Stefanowitsch

\title{
Keine Grammatik ohne Konstruktionen: Ein logisch-ökonomisches Argument für die Konstruktionsgrammatik ${ }^{1}$
}

\begin{abstract}
Es ist unbestritten, dass in allen natürlichen Sprachen nicht-kompositionelle FormBedeutungspaare verschiedener Komplexitäts- und Abstraktionsgrade existieren. Uneinigkeit besteht dagegen bezüglich der Frage, ob diese Form-Bedeutungspaare als Teil der Grammatik oder gar, wie in der Konstruktionsgrammatik postuliert, als grundsätzliches grammatisches Organisationsprinzip zu verstehen sind. In meinem Beitrag argumentiere ich für eine zentrale Rolle von Konstruktionen bei der Repräsentation sprachlichen Wissens: Da Menschen offensichtlich in der Lage sind, Konstruktionen (im oben genannten Sinne) zu erwerben und zu verarbeiten, muss ein entsprechendes, konstruktionsverarbeitendes System existieren. Dieses kann auch nicht-idiomatische (regelhafte) Strukturen verarbeiten. Umgekehrt kann aber ein regelverarbeitendes System nicht ohne Weiteres idiomatische Strukturen verarbeiten. Das Sparsamkeitsprinzip sagt uns, dass eine Grammatik mit nur einem System einer mit zwei Systemen vorzuziehen ist, wenn es keine zwingenden Gründe für die Annahme eines zweiten Systems gibt. Neben diesem logisch-ökonomischen Argument diskutiere ich abschließend die Möglichkeit, anhand der systematischen Verletzung scheinbar allgemeingültiger grammatischer Regeln die Plausibilität eines konstruktionsverarbeitenden Systems empirisch zu untermauern.
\end{abstract}

\section{Einleitung}

Jede grammatiktheoretische Strömung innerhalb der modernen Sprachwissenschaft erkennt die Existenz von nicht-kompositionellen Form-Bedeutungspaaren mit verschiedenen Komplexitäts- und Abstraktionsgraden in allen natürlichen Sprachen an. Diese Form-Bedeutungspaare werden unter einer Vielzahl von Begriffen wie „Phraseologismus“, „Phrasem“, „Phraseolexem", „festes Syntagma“, „(konstruktionelles) Idiom“, „Formel", „Floskel", , ,Redewendung $^{\text {cc }}$, u.a. diskutiert; in der Konstruktionsgrammatik werden sie alle unter dem Begriff der „Konstruktion“ zusammengefasst.

1 Für interessante Fragen und Anregungen danke ich den Teilnehmer/innen der 46. Jahrestagung des Instituts für Deutsche Sprache, insbesondere Heike Behrens, Stefan Engelberg, Joachim Jacobs, Tibor Kiss, Gereon Müller, Beatrice Primus, Peter Uhrig, Gert Webelhuth und Alexander Ziern. Ferner danke ich den Herausgeber/innen für ihre aufmerksame Lektüre des Manuskripts und ihre konstruktive Kritik. Ein besonderer Dank gebührt Juliana Goschler für ausführliche Diskussionen und Verbesserung-svorschläge während der Entstehung dieser Arbeit. 
Uneinigkeit besteht dagegen bezüglich der Frage, welche Rolle diese Konstruktionen in der Grammatik natürlicher Sprachen spielen. In den meisten Theorien geht man davon aus, dass diese Konstruktionen Ausnahmeerscheinungen sind, die außerhalb des eigentlichen, regelgestützten Kerns der Grammatik - etwa im Lexikon - aufgelistet werden müssen. In der Konstruktionsgrammatik werden sie dagegen als grundsätzliches grammatisches Organisationsprinzip verstanden.

Im vorliegenden Beitrag werde ich für eine zentrale Rolle von Konstruktionen bei der Repräsentation sprachlichen Wissens argumentieren. Dabei gehe ich zunächst von einem deduktiven, logisch-ökonomischen Argument aus, das seit den Anfängen der Konstruktionsgrammatik immer wieder mehr oder weniger explizit in die Theoriediskussion eingebracht worden ist, das aber bisher keine ausreichende Beachtung gefunden hat.

Dieses Argument lässt sich etwa wie folgt zusammenfassen: Menschen sind offensichtlich in der Lage, Konstruktionen (im konstruktionsgrammatischen Sinne) zu erwerben und zu verarbeiten; es muss also in den Köpfen der Sprecher/innen ein entsprechendes, konstruktionsverarbeitendes System existieren. ${ }^{2}$ Ein System, das irreguläre und idiomatische Strukturen verarbeiten kann, ist logischerweise auch in der Lage, regelhafte und kompositionelle Strukturen zu verarbeiten, denn letztere unterscheiden sich von ersteren ja nur durch ihre weniger starken syntaktischen und semantischen Beschränkungen. Umgekehrt kann aber ein regelverarbeitendes System nicht ohne weiteres idiomatische Strukturen verarbeiten. Ockhams Rasiermesser sagt uns, dass eine Grammatik mit nur einem System einer mit zwei Systemen ceterus paribus vorzuziehen ist.

Der Beitrag ist wie folgt strukturiert: Im zweiten Unterkapitel stelle ich zunächst in allgemeiner Form die Grundannahmen der Konstruktionsgrammatik dar und diskutiere dann speziell den Kernbegriff der Konstruktion als nicht-kompositionelles Form-Bedeutungspaar und seine potenzielle Reichweite in der Architektur natürlichsprachlicher Grammatiken. Im dritten Unterkapitel folgt eine ausführliche Diskussion des logischen Arguments für die Existenz von Konstruktionen sowie eine Fallstudie des Ausdrucks [WH in aller Welt], die zeigen soll, dass Konstruktionen bis weit in die Bereiche der Grammatik hineinwirken, die traditionell als „Kerngrammatik“" bezeichnet werden. Im vierten Unterkapitel stelle ich das ökonomische Argument für die Konstruktion als einziges Organisationsprinzip natürlicher Sprachen vor und präsentiere eine Fallstudie des grammatischen Musters

Es sei hier dahingestellt, ob es sich bei diesem System um ein sprachspezifisches kognitives „Modul ${ }^{\text {cc }}$ zur Verarbeitung von Konstruktionen handelt, oder um eine allgemeinere kognitive Fähigkeit. In der Konstruktionsgrammatik dürfte derzeit die zweite Möglichkeit eher konsensfähig sein (vgl. z.B. Langacker 1987; Lakoff 1987; Goldberg 2006), aber keines der Kernprinzipien der Konstruktionsgrammatik schließt die erste Möglichkeit kategorisch aus. Für das im Folgenden dargestellte Argument spielt diese Frage keine Rolle: Entscheidend ist nur, dass ein kognitives System existiert, das Konstruktionen verarbeiten kann. 
$\left[\mathrm{NP}_{\mathrm{i}}\right.$ hat POSS $_{\mathrm{i}}$ Preis $]$, die zeigen soll, dass die Verletzung von Regeln Hinweise auf die konstruktionsgestützte Natur unseres sprachlichen Wissens geben kann.

\section{Theoretischer Hintergrund}

\subsection{Grundannahmen der Konstruktionsgrammatik}

Unter der Bezeichnung ,Konstruktionsgrammatik" wird eine recht heterogene Klasse von grammatiktheoretischen Ansätzen zusammengefasst, deren kennzeichnendes Merkmal es ist, dass sie das sprachliche Zeichen im saussureschen Sinne - in der Konstruktionsgrammatik „Konstruktion “ genannt - als zentrales oder sogar einziges Organisationsprinzip natürlichsprachlicher Grammatiken betrachten. Der Zeichenbegriff der Konstruktionsgrammatik geht dabei insofern über das klassische saussuresche Verständnis hinaus, als dass darunter Strukturen mit beliebigem Komplexitäts- und Abstraktionsgrad erfasst werden, solange sie arbiträre, nicht aus ihren Bestandteilen ableitbare Eigenschaften besitzen.

Eine häufig zitierte und in der Konstruktionsgrammatik akzeptierte Definition des Begriffs „Konstruktion“ findet sich in Goldberg (1995):

$C$ ist eine Konstruktion dann und nur dann, wenn $C$ ein Form-Bedeutungspaar $<\mathrm{F}_{i}, \mathrm{~S}_{i}>$ ist, dergestalt, dass irgendein Aspekt von $\mathrm{F}_{i}$ oder irgendein Aspekt von $\mathrm{S}_{i}$ sich nicht auf der Grundlage der Komponenten von $C$ oder von bereits etablierten Konstruktionen vorhersagen lässt. (Goldberg 1995, S. 4, übers. Fischer/Stefanowitsch 2006, S. 5)

Der Begriff „Bedeutung" ist dabei in dieser Definition weit zu fassen, er bezieht sich nicht nur auf wahrheits- oder framesemantische Eigenschaften einer Konstruktion, sondern auch auf Aspekte des Gebrauchs wie Diskursfunktion, Informationsstruktur usw. ${ }^{3}$

Die Konstruktionsgrammatik geht davon aus, dass sprachliches Wissen vollständig in Form von Konstruktionen von unterschiedlicher Komplexität und unterschiedlichem Abstraktionsgrad repräsentiert ist (die Gesamtheit aller Konstruktionen einer Sprache wird dabei als „Konstruktikon “ bezeichnet). Anders als Kritiker der Konstruktionsgrammatik manchmal behaupten (z.B. Leiss 2009), bedeutet das aber nicht, dass das sprachliche Wissen als unstrukturierte Ansammlung strukturloser sprachlicher Zeichen verstanden werden soll. Konstruktionen sind sowohl intern als auch in Bezug zueinander strukturiert, sodass regelhafte Aspekte einer Konstruktion auf einer höheren Abstraktionsebene repräsentiert sind und von

Eine seltener zitierte, aber nicht weniger akzeptierte Definition in Lakoff (1987) macht dies explizit: „Eine Konstruktion sei ein Form-Bedeutungspaar (F, M), wobei F eine Menge von Bedingungen bezüglich der syntaktischen und phonologischen Form und M eine Menge von Bedingungen bezüglich Bedeutung und Vervendung ist" (Lakoff 1987, S. 467, übers. Fischer/Stefanowitsch 2006, S. 5, eigene Hervorhebung). 
mehreren Konstruktionen geteilt werden können. ${ }^{4}$ Auf der höchsten Abstraktionsebene finden sich dann Konstruktionen, die traditionellen Regeln ähneln (etwa die Subjekt-Prädikat-Konstruktion oder die Modifikator-Kopf-Konstruktion, siehe unten). Die Form und die Bedeutung von Konstruktionen ist also systemintern durch Verknüpfungen mit Konstruktionen ähnlicher Form und Bedeutung motiviert; sie ist zudem beschränkt durch allgemeine Eigenschaften des kognitiven Systems (vgl. z.B. Langacker 1987) und durch die kommunikativen Funktionen, die die Konstruktionen erfüllen müssen.

Über diese gemeinsamen Grundannahmen hinaus unterscheiden sich konstruktionsgrammatische Theorien teilweise drastisch voneinander (eine Diskussion der wichtigsten Unterschiede und Gemeinsamkeiten findet sich in Fischer/Stefanowitsch 2006). Im Rahmen der vorliegenden Arbeit spielen die meisten dieser Unterschiede keine große Rolle, da es hier um die grundsätzliche Frage gehen soll, ob und in welchem Umfang es plausibel ist, Konstruktionen als Teil des sprachlichen Wissens zu betrachten.

Ein entscheidender Unterschied muss aber noch erwähnt werden: Einige Versionen der Konstruktionsgrammatik betrachten das Kriterium der Nicht-Kompositionalität nicht mehr als notwendige Voraussetzung, um einer Struktur Konstruktionsstatus zuzuschreiben. Alternativ legen sie eine Art allgemeines Repräsentationskriterium zugrunde:

Als Konstruktion wird jedes sprachliche Muster akzeptiert, wenn irgendein Aspekt seiner Form oder Funktion sich auf der Grundlage seiner Komponenten oder anderer, bereits bekannter Konstruktionen nicht genau vorhersagen lässt. Außerdem werden auch Muster, die voll vorhersagbar sind, als Konstruktionen gespeichert, wenn sie ausreichend bäufgg sind. (Goldberg 2006, S. 5, übers. Stefanowitsch, eigene Hervorhebung)

Dieser Konstruktionsbegriff stammt aus einer Strömung innerhalb der Kognitiven Linguistik, die als ,gebrauchsgestütztes Modell“ (Usage-Based Model) bekannt ist und die die Konventionalität sprachlicher Zeichen in den Mittelpunkt stellt (vgl. Langacker 1987). Die Idee, dass häufige Strukturen auch dann in ihrer Gesamtheit gespeichert werden (können), wenn sie voll regelhaft und kompositionell sind, ist natürlich grundsätzlich mit der Idee eines strukturierten Konstruktikons vereinbar. Es ist nachvollziehbar, dass die hohe Gebrauchshäufigkeit einer sprachlichen Struktur dazu führt, dass diese Struktur mental als Einheit repräsentiert und in der Sprachverarbeitung nicht mehr für jedes Gebrauchsereignis aus seinen Einzelteilen zusammengesetzt bzw. in diese zerlegt wird. Trotzdem sollten die zwei Arten mentaler Einheiten terminologisch unterschieden werden. In Stefanowitsch (2009) schlage ich vor, den Konstruktionsbegriff für syntaktisch und/oder semantisch nicht vorhersagbare Strukturen zu verwenden, und

\footnotetext{
$4 \quad$ Vgl. z.B. Kay (1997, S. 129), der als Beispiel die englische „Left-Isolation Construction“ nennt, die von allen Konstruktionen geteilt wird, bei denen eine Konstituente nicht in ihrer kanonischen Position, sondern am linken Rand des Satzes steht, also etwa bei $W h$-Sätzen und verschiedenen Arten von Topikalisierungsstrukturen.
} 
Strukturen, die (z.B. aufgrund ihrer Häufigkeit) in ihrer Gesamtheit repräsentiert werden, als ,Muster" zu bezeichnen. Es ist dann klar, dass alle Konstruktionen auch Muster sind, nicht aber umgekehrt.

\subsection{Reichweite des Konstruktionsbegriffs}

Eine Konstruktion ist also jede sprachliche Struktur in einer Sprache, deren formale oder funktionale Eigenschaften sich nicht auf der Grundlage bereits vorhandener Konstruktionen in dieser Sprache vorhersagen lassen.

Dazu gehören zunächst natürlich monomorphemische Wörter und Flexions- und Derivationsmorpheme wie trink-/,,Flüssigkeit in den Mund nehmen und schlucken " oder $\left[\mathrm{V}_{\mathrm{x}}\right.$-er $] /$,jemand, der regelmäßig $\mathrm{X}$ tut"; Morpheme sind ja dadurch charakterisiert, dass sie nicht-vorhersagbare Form-Bedeutungspaare sind.

Aber auch morphologisch komplexe Wörter können Konstruktionen in diesem Sinn sein, sofern sich ihre Form oder Bedeutung nicht aus ihren Einzelteilen vorhersagen lässt. Kombiniert man z.B. trink- und $\left[\mathrm{V}_{\mathrm{X}}-\mathrm{er}\right]$, so lässt sich die Form Trinker vorhersagen. Auf der Bedeutungsseite ergibt sich dagegen die erwartete Bedeutung, ,jemand, der Flüssigkeit in den Mund nimmt und schluckt", die nur einen Teil der tatsächlichen Bedeutung des Wortes erfasst: Ein Trinker ist, ,jemand, der regelmäßig und qwanghaft alkoholhaltige Flüssigkeit in den Mund nimmt und schluckt". Da die kursiv gesetzten zusätzlichen Bedeutungskomponenten nicht vorhersagbar sind, muss auch das Wort Trinker als Konstruktion betrachtet werden.

Umgekehrt ist zum Beispiel die Bedeutung der im Deutschen Referenzkorpus des Instituts für Deutsche Sprache belegten Komposita Apfelgetränk, Orangengetränk, Beerengetränk, Erdbeergetränk, Hafergetränk und Gerstengetränk vorhersagbar. Auf der Formseite unterscheiden sich die Wörter dagegen auf unvorhersagbare Weise. So steht beim Apfelgetränk das Erstglied im Singular und zwischen den Kompositionsgliedern steht kein Fugenelement; beim Orangengetränk, und beim Beerengetränk, dagegen steht das Erstglied im Plural (bzw. die Kompositionsglieder sind durch ein Fugen- $n$ verbunden). Bei einem Getränk aus Erdbeeren würde man entweder das Wort *Erdbeeregetränk (analog zu Apfelgetränk) oder das Wort *Erdbeerengetränk (analog zu Orangengetränk) erwarten, stattdessen findet sich im Deutschen Referenzkorpus ausschließlich das Wort Erdbeergetränk mit dem auf Komposita beschränkten Erstglied Erdbeer, und auch eine Google-Suche fördert keine Treffer für die anderen beiden Formen zutage, die eindeutig deutschen Muttersprachlern zuzuordnen sind. Schließlich muss beim nicht-zählbaren Erstglied Gerste ein Fugen- $n$ stehen, während das ebenfalls nicht-zählbare Erstglied Hafer kein Fugenelement benötigt. ${ }^{5}$

Die Erstglieder verhalten sich nicht nur in Komposition mit dem Zweitglied Getränk so, wie hier dargestellt, sondern typischerweise in allen Komposita. Das ändert aber nichts an der 
An dieser Stelle lässt sich der Unterschied zwischen Konstruktionen und Mustern demonstrieren. Das Wort Esser (,,jemand, der isst ${ }^{c}$ ) lässt sich, anders als das Wort Trinker, sowohl in seiner Form als auch in seiner Bedeutung vorhersagen. Trotzdem ist die Annahme gerechtfertigt, dass das Wort im mentalen Lexikon deutscher Muttersprachler als Muster repräsentiert ist und nicht jedes Mal aus seinen Bestandteilen erzeugt wird. Dafür spricht unter anderem, dass es konventionalisierte Kollokationen mit diesem Wort gibt (ein guter/schlechter Esser ist ein konventioneller Ausdruck, ein guter/schlechter Essender nicht; gleiches gilt für ein Esser mehr/ qu viel im Vergleich zu ein Essender mehr/ zu viel).

Dass einzelne Morpheme ebenso wie formal oder semantisch unregelmäßige komplexe Wörter der oben zitierten Konstruktionsdefinition entsprechen, dürfte unstrittig sein, sie werden in allen Theorien im Lexikon verortet. Dass sie als „Konstruktionen " bezeichnet werden, mag ungewohnt sein, ist aber eine oberflächliche terminologische Frage (und nicht alle konstruktionsgrammatischen Ansätze folgen dieser Terminologie ${ }^{6}$ ).

Es dürfte auch weitgehend Einigkeit darüber bestehen, dass der Begriff der Konstruktion auf Syntagmen mit nicht vorhersagbaren formalen oder semantischen Eigenschaften anwendbar ist, wie z.B. jmd. unter den Tisch trinken oder einen über den Durst trinken. Semantisch unvorhersagbare Eigenschaften dieser Syntagmen sind z.B., dass sie (wie auch das Kompositum Trinker) auf die Einnahme alkoholischer Getränke beschränkt sind, dass jemand, der unter den Tisch getrunken worden ist, nicht tatsächlich unter dem Tisch liegen muss, und dass jemand, der einen über den Durst getrunken hat, immer noch Durst haben kann. Formal unvorhersagbar ist, dass die sprachliche Flexibilität dieser Syntagmen eingeschränkt ist, man also z.B. nicht * jmd. unter den Stubl trinken oder *einen unter den/dem Durst trinken kann. Problematisch ist an solchen Syntagmen höchstens die Tatsache, dass sie partiell semantisch transparent und partiell syntaktisch flexibel sind, dass sie also in einer Form im Lexikon repräsentiert werden müssen, die allgemeinen Regeln in beschränktem Umfang zugänglich ist, doch entsprechende Lösungen existieren in verschiedenen regelgestützten Theorien (vgl. z.B. Tree-Adjoining Grammar, Abeillé/Schabes 1989; HPSG (Head-driven Phrase Structure Grammar), Sailer 2003).

Die Einigkeit bröckelt vermutlich bei Ausdrücken wie anf NP trinken, die variable Leerstellen enthalten, in die jeder formal passende Ausdruck eingesetzt werden kann (ein weiteres Beispiel wären die modalen Infinitive im Deutschen, vgl. Stefanowitsch 2006, 2010); sie endet spätestens bei Kon-

\footnotetext{
Tatsache, dass es sich um nicht-vorhersagbare Formen handelt - die Nicht-Vorhersagbarkeit ist lediglich auf einer allgemeineren Ebene anzusiedeln.

Siehe z.B. Langacker (1987), der das, was in anderen konstruktionsgrammatischen Ansätzen „Konstruktion" heißt, als „symbolische Einheit" (symbolic unit) bezeichnet und den Begriff "Konstruktion" (construction) auf komplexe Strukturen aus mindestens zwei solchen symbolischen Einheiten beschränkt.
} 
figurationen abstrakter grammatischer Kategorien, die lexikalisch nicht mehr spezifiziert sind, wie etwa Resultativen (vgl. z.B. Müller 2006), verblosen Direktiven (Jacobs 2008) oder Relativsätzen (Webelhuth in diesem Band). In der Konstruktionsgrammatik werden auch solche Strukturen als Konstruktionen verstanden; ihnen gilt das Hauptinteresse konstruktionsgrammatischer Theoriebildung. Ihr Status ergibt sich aus den oben zitierten Definitionen: Es handelt sich um Form-Bedeutungspaare, die nicht-vorhersagbare formale oder semantische Eigenschaften haben.

Nehmen wir den Fall der Resultativkonstruktion, die sich in Beispielen wie den folgenden manifestiert:
a. Sie trank ihren Calvados leer. [WAM]
b. Norina trank ihr Glas leer. [M02]
c. [Er] trank die Minibar leer. [RHZ02]

Bei (1a) mag es zunächst aussehen, als handle es sich um die regelhafte Erweiterung des transitiven Satzes (2a) um eine Adjunkt-AP leer:

(2) a. Sie trank ihren Calvados.

b. ? Norina trank ihr Glas (vgl. [Sie machten] an jeder Gastwirtschaft stopp ... und tranken ein Gläschen. [RHZ04])

c. *Er trank die Minibar.

Diese Analyse ist bei (1b) schon problematischer, da der Status des dafür notwendigen transitiven (2b) zweifelhaft ist. Bei (1c) schließlich ist die Analyse ausgeschlossen, da der Satz ungrammatisch ist. Ebenso ist es problematisch, dass bei (1b-c) tatsächlich das Glas bzw. die Minibarleer ist, bei (1a) der Calvados jedoch nicht leer, sondern nicht mehr da wäre. Schließlich bliebe unklar, woher die resultative Lesart kommt; sie ergibt sich keinesfalls zwingend aus dem Anfügen einer AP an einen transitiven Satz, wie die nichtresultativen Lesarten von Sätzen wie Sie trank ihren Calvados eiskalt oder Sie trank ihren Calvados pur zeigen.

Stefan Müller (2006) zeigt im Rahmen einer HPSG-Analyse, dass diese Strukturen durch eine Lexikonregel erzeugt werden können, die die beobachtete Valenz erzeugen und gleichzeitig eine resultative Lesart hinzufügen kann. Dass Müller von „Regeln“" spricht, darf hier nicht darüber hinwegtäuschen, dass diese unter den oben genannten Konstruktionsbegriff fallen: Lexikonregeln in der HPSG sind formale Operationen, die einen ohne die Regel nicht vorhersagbaren semantischen Effekt haben können; wo dies der Fall ist, handelt es sich um Form-Bedeutungspaare im Sinne des oben genannten Konstruktionsbegriffs.

Konstruktionsgestützte Analysen solcher abstrakten grammatischen Strukturen werden von Gegnern der Konstruktionsgrammatik häufig mit dem Argument angegriffen, man könne sie durchaus aus allgemeinen (möglicherweise sogar universellen) Regeln ableiten. So leitet Gereon Müller (in diesem Band) verblose Direktive (Zur Hölle mit dieser Regierung, In den Müll mit 
diesen Klamotten) aus Regeln ab, die in anderen Sprachen für Antipassivbildung und stilistische Voranstellung zuständig sind. Solche Analysen sind interessant (ob man ihnen im Einzelnen folgen mag oder nicht), aber als Gegenargumente einer konstruktionsgrammatischen Perspektive werden sie erst dann relevant, wenn sich zeigen lässt, dass sich auch ihre Bedeutung aus der Anwendung allgemeiner Regeln ableiten lässt. Dies ist im Allgemeinen nicht der Fall; Müller gelingt seine Herleitung der verblosen Direktiven nur deshalb, weil er deren semantische Eigenschaften außer Acht lässt. Damit zeigt er im besten Fall, dass die Form von verblosen Direktiven sich innerhalb des in natürlichen Sprachen möglichen Formeninventars bewegt, nicht aber, dass die Form verbloser Direktive vorhersagbar oder deren Bedeutung aus ihrer Form ableitbar wäre. Auch unter seiner Analyse bleiben sie Konstruktionen.

Abschließend sei darauf hingewiesen, dass in der Konstruktionsgrammatik konsequent auch die abstraktesten grammatischen Strukturen als Konstruktionen analysiert werden; Beispiele sind die englischen Argumentstrukturkonstruktionen (Goldberg 1995), die Subjekt-Prädikat-Konstruktion (Kay/Fillmore 1999) und die deutsche Verberst-Konstruktion (Diessel 1997).

\subsection{Regeln vs. Konstruktionen}

Wie in der Diskussion der Resultativkonstruktion bereits angedeutet, charakterisiert der Konstruktionsbegriff bestimmte Eigenschaften sprachlichen Wissens, er sagt jedoch für sich genommen weder etwas darüber aus, wie dieses sprachliche Wissen mental repräsentiert sein könnte, noch darüber, wie es am besten darzustellen ist.

Natürlich lässt sich jede Konstruktion im konstruktionsgrammatischen Sinne auch als Regel darstellen, sofern man ein Modell annimmt, in dem Regeln semantische und pragmatische Informationen enthalten dürfen; umgekehrt lässt sich auch eine rein formale Regel als Konstruktion darstellen, sofern man ein Modell annimmt, in dem die Bedeutungsseite einer Konstruktion leer bleiben darf (so wie in Langackers Kognitiver Grammatik). Die Argumentstrukturkonstruktionen in Goldberg (1995) lassen sich z.B. als lexikalische Regeln darstellen und die lexikalischen Regeln in Müller (2006) als Konstruktionen, ohne dass allein durch die Darstellung eine grundsätzlich andere Art sprachlichen Wissens angenommen würde. Im Rahmen einer theoriebildenden Diskussion ist jedoch eben die Frage entscheidend, welche Art von sprachlichem Wissen in einer solchen Konstruktion oder Regel erfasst werden darf: Die Unterschiede zwischen den Theorien ergeben sich aus der Antwort auf diese Frage (und natürlich aus der Gesamtarchitektur, in die die ,Regeln" oder „Konstruktionen" eingebettet sind). ${ }^{\text {? }}$

Vgl. in diesem Zusammenhang Jacobs (2009), der darauf hinweist, dass die Unterschiede zwischen konstruktionistischen und projektionistischen Analysen in vielen Fällen nur durch 
Mangelnde Klarheit über den Unterschied zwischen Notation und theoretischer Substanz kann leicht dazu führen, dass die Darstellung von Konstruktionen als Regel fälschlicherweise als ein Gegenargument zu einer konstruktionsgestützten Analyse oder gar als Beweis der Nichtexistenz von Konstruktionen gewertet wird. Wenn eine „Regel“ ein Form-Bedeutungspaar spezifiziert, ist sie eine Konstruktion, deren Notation manipuliert wurde, um sie wie eine Regel aussehen zu lassen. Eine solche „Regel mag in der Darstellung elegant und nützlich sein, aber auf ihrer Grundlage lässt sich der philosophische Überbau der generativen Grammatik Chomskyschen Zuschnitts (etwa die Idee einer wie auch immer gearteten Universalgrammatik) nicht rechtfertigen.

\section{Das logische Argument: Konstruktionen als Teil der Sprachkompetenz}

Akzeptiert man die Existenz nicht-kompositioneller und/oder formell idiosynkratischer sprachlicher Strukturen, so lässt sich folgendes logisches Argument für die Existenz eines - wie auch immer gearteten - konstruktionsverarbeitenden Systems konstruieren:

Alle Sprachen enthalten nicht-kompositionelle und/oder syntaktisch nicht voll regelhafte Strukturen.

Sprecher können diese Strukturen produzieren und verarbeiten.

Daraus folgt: Es existiert ein System, das nicht-kompositionelle und/ oder syntaktisch nicht voll regelhafte Strukturen repräsentieren und zur Verarbeitung bereitstellen kann.

Das logische Argument besagt zunächst nur, dass es keine mentale Grammatik geben kann, die nicht auch Konstruktionen verarbeiten kann, oder die durch ein entsprechendes System ergänzt wird. Wie in Abschnitt 2.2 oben dargestellt, ist dies im Falle von Wörtern und idiomatischen Redewendungen auch relativ unstrittig und führt dazu, dass Wörter und idiomatische Syntagmen im Lexikon verortet werden und die syntaktische Komponente einzig für kerngrammatische Strukturen zuständig ist.

Das Problem an dieser Sichtweise ist allerdings, dass idiomatische Syntagmen häufig eng mit scheinbar kerngrammatischen Strukturen und Regeln interagieren. Das hat Konsequenzen für die Plausibilität einer Trennung von Kerngrammatik und Lexikon (Fillmore 1985). Bevor ich diese Konsequenzen diskutiere, möchte ich die Interaktion von Konstruktionen mit kerngrammatischen Regeln am Beispiel eines deutschen Ausdrucks zeigen,

die Darstellung bedingt sind, und dass konstruktionistische Analysen auf Phänomene begrenzt werden sollten, in denen eine projektionistische Analyse unmöglich oder zumindest unmotiviert ist. 
dessen englisches Äquivalent Charles Fillmore in seiner ersten Arbeit zur Konstruktionsgrammatik (Fillmore 1985) kurz anreißt. Dieser Ausdruck besteht aus einem Interrogativpronomen gefolgt von der PP in aller Welt:
a. Was in aller Welt will Frau Merkel erreichen, ist sie völlig naiv und vergesslich (Stichwort Kosovo)? [NUZ08]
b. Warum in aller Welt sollte man ein Rockkonzert besuchen? Die Luft ist schlecht, der Sound meist ebenso. [NUZ07]
c. Inzenberg, wo in aller Welt liegt das denn? [A97]
d. Aber wie in aller Welt wird es ausgesprochen? [R97]

In Wörterbüchern wird die Bedeutung dieses Ausdrucks mit „Steigerungs-

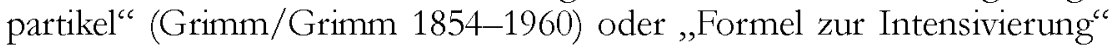
(Wahrig-Burfeind (Hg.) 2006) angegeben. Die Beispiele in (3) zeigen aber, dass damit nur ein Teil der Bedeutung erfasst ist; zusätzlich drückt das Idiom Unverständnis des Sprechers bezüglich der Proposition des Satzes aus, in dem sie auftritt. Weder die Intensivierung noch der Ausdruck von Unverständnis ergeben sich kompositionell aus der Bedeutung der einzelnen Konstituenten des Ausdrucks oder lassen sich aus allgemeinen pragmatischen Prinzipien herleiten, wie eine Ersetzung durch synonyme Wörter und Ausdrücke zeigt:
a. *Was überall will Frau Merkel erreichen?
b. *Was in/ auf der ganzen Welt will Frau Merkel erreichen?
c. *Was in allen Ländern der Erde will Frau Merkel erreichen?

Die Sätze in $(4 a-c)$ sind zunächst ungrammatisch, da keiner der kursiv gesetzten Ausdrücke direkt hinter dem $W h$-Pronomen stehen kann. Selbst, wenn man dies ignoriert, hätten $(4 a-c)$ aber nicht dieselbe Bedeutung wie die entsprechenden Sätze mit in aller Welt, sondern könnten nur wörtlich interpretiert werden. Damit fällt der Ausdruck [WH in aller Welt] aus semantischen und formalen Gründen unter den konstruktionsgrammatischen Konstruktionsbegriff; ich werde ihn im Folgenden als WhIAW-Konstruktion oder kurz als WhIAW bezeichnen.

Die WhIAW-Konstruktion gehört zu einer Klasse von formal ähnlichen Konstruktionen, die alle eine intensivierende Bedeutung haben, die sich aber darüber hinaus semantisch klar unterscheiden und die auch bezüglich ihres syntaktischen Verhaltens keine homogene Gruppe bilden; zu dieser Gruppe gehören z.B. WH vur Hölle, WH rum Teufel, WH um Himmelswillen, WH in Dreiteufelsnamen u.a. Um in jedem Falle entscheiden zu können, ob eine bestimmte formale oder semantische Eigenschaft spezifisch für WhIAW ist, oder ob sie für all diese intensivierenden PPs gilt, müsste man jeden dieser Ausdrücke detailliert analysieren; dies kann hier aber nicht geleistet werden, sodass ich im Folgenden nur punktuell auf Unterschiede zwischen WhIAW und anderen Ausdrücken eingehen werde. Dies birgt das 
Risiko einer Zuschreibung von Eigenschaften an die WhIAW-Konstruktion, die besser bei allgemeineren Konstruktionen verortet wären; das ändert aber nicht grundsätzlich etwas an der konstruktionellen Natur der betreffenden Eigenschaften. ${ }^{8}$

Die WhIAW-Konstruktion kann nicht nur in interrogativen Hauptsätzen stehen, sondern auch in indirekten Fragesätzen als Komplement von Verben und Substantiven: ${ }^{9}$

a. Jeder andere würde sich an seiner Stelle fragen, wo in aller Welt er jetzt noch Freunde suchen soll. [NUN96]

b. Zudem werfen sie die Frage auf, was in aller Welt der Wiener Exzentriker [Falco] mit Wolfgang Amadeus Mozart gemeinsam haben soll. [HZ04]

Im Deutschen Referenzkorpus kommt WhIAW in indirekten Fragesätzen mit den Verben und Verbkomplexen in (6a) und den Substantiven in (6b) vor:

(6) a. fragen (9-mal), wissen (2-mal), sich fragen, dariber nachdenken, hinterfragen, rätseln, überlegen, sagen, erklären (je 1-mal)

b. Frage (9-mal), Bemerkung (1-mal)

Die Verwendung von WhIAW in indirekten Fragesätzen unterliegt dabei subtilen Beschränkungen, bei denen es zunächst so scheinen mag, als ob sie lexikalischer Natur seien. Tatsächlich aber lassen sie sich alle auf die folgende allgemein semantisch-pragmatische Beschränkung zurückführen: ${ }^{10}$

So ließe sich beispielsweise einwenden, dass die Position von in aller Welt direkt hinter dem Wh-Pronomen nicht unvorhersagbar ist, da intensivierende PPs grundsätzlich in dieser Position stehen können. Dies ließe sich durch eine allgemeinere Regel erfassen, die besagen würde, dass $w$ h-Pronomen durch intensivierende PPs (und nur durch diese) modifiziert werden dürfen. Diese „Regel“ müsste aber Bezug auf die Bedeutung der PP nehmen (denn nur so lässt sich feststellen, ob diese ,intensivierend" ist) und wäre somit selbst eine Konstruktion im hier angenommenen Sinne. Interessant ist aber, dass in aller Welt, anders als alle anderen intensivierenden PPs, nahezu kategorisch auf diese Position beschränkt ist (siehe unten); dies ließe sich nicht aus einer allgemeinen Konstruktion $\left[\mathrm{Wh}\left[\mathrm{PP}_{\text {intens }}\right]\right]$ herleiten, müsste also doch auf der Ebene der WhIAW-Konstruktion spezifiziert werden.

9 Außerdem kann WhIAW auch in $W b$-Verbletzt-Sätzen auftreten; das Deutsche Referenzkorpus enthält zwar kein entsprechendes Beispiel, aber Beispiele wie Was in aller Welt Falco wobl mit Mogart zu tun hat? scheinen akzeptabel.

10 Diese Beschränkung ist nicht so zu verstehen, dass WhIAW nur in Sätzen auftreten kann, die zum Vollzug des illokutionären Akts einer Frage dienen - es muss keine Aufforderung vorliegen, die offene Frage im Kopf des Sprechers oder des Referenten des Matrixsatzes zu beantworten. Die Beispiele in $(9 a-c)$ unten zeigen dies: Keins von ihnen stellt direkt oder indirekt eine solche Aufforderung dar. Man kann die Beschränkung in (7) deshalb nicht ohne Weiteres mit der Unterscheidung zwischen Fragesemantik und Fragepragmatik erfassen, die vor allem in der klassischen generativen Grammatik häufig getroffen wird (vgl. z.B. Holler 2007 für eine zusammenfassende Darstellung dieser Unterscheidung). 
(7) WhIAW kann nur verwendet werden, wenn das $W$-Pronomen Teil eines Satzes ist, dessen Proposition als unbeantwortete Frage im Kopf des Sprechers oder des Referenten eines Arguments im Matrixsatz interpretiert werden muss.

Diese Beschränkung erklärt zunächst natürlich, warum WhIAW in interrogativen Hauptsätzen auftritt, denn diese stellen ihre Proposition ja direkt als offene Frage dar. Sie erklärt auch, warum WhIAW in indirekten Fragesätzen mit Abstand am häufigsten mit den Köpfen Frage und (sich) fragen auftritt, und auch das Vorkommen mit den Verben sich fragen, dariber nachdenken, hinterfragen, rätseln und überlegen ergibt sich daraus.

Das Vorkommen mit den Verben wissen, sagen und erklären sowie mit dem Substantiv Bemerkung scheint die Beschränkung dagegen zu verletzen, und tatsächlich ist die Verwendung von WhIAW mit diesen und ähnlichen Verba Dicendi und Sentiendi nicht ohne Weiteres akzeptabel:
a. *Maria sagte, was in aller Welt Falco mit Mozart zu tun hatte.
b. *Maria erklärte Hans, was in aller Welt Falco mit Mozart zu tun hatte.
c. *Maria musste, was in aller Welt Falco mit Mozart zu tun hatte.

Das ändert sich aber in Kontexten, in denen die Verwendungsweise des jeweiligen Verbs voraussetzt, dass eine unbeantwortete Frage im Kopf des Sprechers oder eines der Referenten des Matrixsatzes existiert; auf diese Weise erklären sich die Vorkommen im Deutschen Referenzkorpus:

(9) a. Wer kann sagen, warum in aller Welt ein Eisenbahntunnel schädlich für ein Gebirge sein soll ... [N98]

b. [Ich] grübelte, wie ich meinen Eltern erkläre, warum in aller Welt ich eine hundsmiserable Note mit nach Hause gebracht hatte. [BRZ07]

c. Doch die Tatsache, dass bisher nur ein paar Insider wissen, wer in aller Welt denn bitteschön Füsun ist, könnte sich bald ändern ... [NUZ08]

Die Phrase wer kann sagen in (9a) zeigt, dass die anschließende Frage aus Sicht des Sprechers unbeantwortet (und unbeantwortbar) ist, und die Verwendung von erklären in (9b) setzt voraus, dass die erwähnten Eltern des Sprechers eine entsprechende Frage im Kopf haben werden, wenn der Sohn ihnen von der schlechten Note erzählt. Das Beispiel in (9c) ist komplexer: Hier werden diejenigen, die eine entsprechende Frage haben, nicht direkt genannt, sondern bilden die nicht-genannte Kontrastmenge zu den genannten Insidern. ${ }^{11}$

\footnotetext{
Die Verwendung von WhIAW mit diesen Verben wird auch dann akzeptabel, wenn diese in Interrogativ- oder Imperativsätzen auftreten, die eine Frage im Kopf des Sprechers voraussetzen, vgl. Weißt du/Sagst du mir bitte/Erklär mir, was in aller Welt Falco mit Mozart qu tun bat.
} 
Abschließend sei darauf hingewiesen, dass die Interpretation eines Satzes die Existenz einer „offenen Frage" zwingend voraussetzen muss, damit WhIAW verwendet werden kann; es genügt nicht, dass ein Verb eine kommunikative Handlung oder einen mentalen Zustand bezeichnet, der eine solche Frage voraussetzen kann. Dies erklärt Unterschiede zwischen semantisch ähnlichen Verben wie denen in $(10 \mathrm{a}-\mathrm{b})$ und $(11 \mathrm{a}-\mathrm{b})$ :

a. Hans rätselte, was in aller Welt Falco mit Mozart zu tun haben könnte.

b. *Hans riet, was in aller Welt Falco mit Mozart zu tun haben könnte.

(11) a. Maria iiberlegte, was in aller Welt Falco mit Mozart zu tun haben könnte.

b. *Maria erforschte/ untersuchte, was in aller Welt Falco mit Mozart zu tun haben könnte.

Das Verb rätseln impliziert semantisch, dass sein Agens eine offene Frage im Kopf hat, das Verb raten tut dies nicht (vgl. 12a); ebenso impliziert überlegen eine solche Frage semantisch, während ähnliche Verben wie erforschen und untersuchen dies nicht tun (vgl. 12b):

(12) a. Hans riet/*rätselte, was Falco mit Mozart zu tun haben könnte, ohne darüber nachzudenken.

b. Maria erforschte/ mtersuchte/*überlegte, was Falco mit Mozart zu tun haben könnte, ohne darüber nachzudenken.

Wenn Verben wie raten, überlegen und erforschen in einem Satzzusammenhang auftreten, in dem die offene Frage im Kopf eines Partizipanten des Matrixsatzes durch andere Mittel zu einem zwingenden Teil der Bedeutung gemacht wird, können sie auch mit WhIAW verwendet werden:

a. Hans versuchte wieder und wieder zu raten, was in aller Welt Falco mit Mozart zu tun haben könnte.

b. Maria untersuchte voller Neugier, was in aller Welt Falco mit Mozart zu tun haben könnte.

Alle scheinbaren verbspezifischen Unregelmäßigkeiten bei der Verwendung von WhIAW in indirekten Fragesätzen ergeben sich also aus der allgemeinen Beschränkung in (7). Die Beschränkung selbst ergibt sich nicht unmittelbar aus der intensivierenden oder Unverständnis anzeigenden Funktion der Konstruktion, muss also als Teil der Konstruktionsbedeutung postuliert werden.

Auch formal hat WhIAW eine Reihe von Eigenschaften, die sich nicht aus allgemeinen Regeln ableiten lassen und die auch nicht durch die Funktion der Konstruktion motiviert sind. Die auffälligste Eigenschaft ist dabei 
die Position der PP in aller Welt, die gemeinsam mit dem Wh-Pronomen eine NP zu bilden scheint. Dies ist deshalb unerwartet, weil - wie eingangs angedeutet - PPs normalerweise in dieser Position nicht stehen können: ${ }^{12}$
a. *Was im Briefkasten ist denn das?
b. Was ist denn das im Briefkasten?

Aus der Funktion „Intensivierung und Unverständnis" lässt sich das Auftreten der PP in dieser Position nicht erklären, denn dann müssten auch andere Adverbiale mit einer solchen Funktion dort stehen können. Dies ist aber nicht der Fall:
a. *Was schon wreder ist denn das?
b. Was ist denn das schon wieder?

Das Auftreten der PP in aller Welt ließe sich auf der Grundlage allgemeiner Regeln nur dann vorhersagen, wenn man sie als Apposition analysieren würde. Für eine solche Analyse würde oberflächlich sprechen, dass diese PP im Schriftdeutschen manchmal durch Kommata vom $W$-Pronomen abgesetzt wird (vgl. 16a), wobei das allerdings in der großen Mehrzahl der Belege nicht der Fall ist (vgl. 16b):
a. Was, in aller Welt, soll das sein? [RHZ97]
b. Was in aller Welt soll das alles? [SOZ06]

Gegen eine Analyse der PP als appositiv spricht aber auch, dass ihr klare Beispiele von Appositionen immer folgen (vgl. 17a). Wenn es sich um eine appositive PP handeln würde, müsste sie auch vor dem $W$ b-Pronomen oder hinter einer weiteren Apposition stehen können, doch das ist - anders als bei der semantisch und strukturell ähnlichen PP rum Teufel - nicht der Fall (vgl. 17b-c):

a. Was in aller Welt, mag man sich fragen, hat Freud mit der ,Analytischen Philosophie des Geistes" zu tun? [R97]

b. *In aller Welt, was hat Freud mit ... zu tun? (vgl. Zum Teufel, warum ist sie ausgerechnet hierher gekommen? [MK2])

c. *Was, mag man sich fragen, in aller Welt, hat Freud mit ... zu tun?

Ein weiteres Argument gegen eine Analyse als Apposition besteht darin, dass in aller Welt - wiederum anders als z.B. ₹um Teufel - nur in Wh-Interrogativsätzen auftreten kann: ${ }^{13}$

\footnotetext{
12 Es gibt noch andere PPs, die in dieser Position stehen können, z.B. von NP (WVer von den beiden liggt?) oder an NP (Was an dem Film bat dir am besten gefallen?) (vgl. Holler 2007, Kap. 4.5.2). Allerdings können diese PPs, anders als in aller Welt, auch in den kanonischen PP-Positionen stehen (Wer ligt von den beiden? Was bat dir an dem Film am besten gefallen?).

13 Betrachtet man die sprachgeschichtliche Entwicklung dieser Ausdrücke, so stellt man fest, dass auch in aller Welt ursprünglich syntaktisch noch relativ frei auftritt. So finden sich noch
} 
(18) a. Wenigstens dann, zum Teufel/*in aller Welt, müssen doch die Emotionen endlich hochkochen! [RHZ04]

b. Javitt, zum Teufel/*in aller Welt, melde dich! [MK2]

Eine Analyse als Apposition ist damit ausgeschlossen. Dagegen lassen sich vereinzelte Beispiele finden, in denen die PP in aller Welt in kanonischen PP-Positionen steht:

a. Jetzt wirst Du mich wieder fragen: was mich denn in aller Welt dazu getrieben hätte, diese merkwürdige Wallfahrt anzutreten? [N94]

b. ... und das Problem erhob sich für die fleißigen Kameraleute: Was tun damit in aller Welt? [M95]

In einem entsprechenden Grammatikmodell ließe sich also argumentieren, dass die PP in aller Welt in einer kanonischen PP-Position generiert und dann durch eine Bewegungsregel in ihre typische Position direkt hinter dem $W$ hPronomen bewegt wird. Dagegen spräche die Tatsache, dass Beispiele wie $(19 a-b)$ extrem selten sind (es sind die einzigen Beispiele ihrer Art im Deutschen Referenzkorpus). Außerdem müsste man eine solche Regel speziell für die PP in aller Welt postulieren, denn schon ähnliche PPs wie qum Teufel und um Himmelswillen verhalten sich anders. Eine solche Regel könnte also bestenfalls einen Mechanismus zur Verfügung stellen, der die PP an ihre typische Position in der Oberflächenstruktur bringt; sie würde nichts daran ändern, dass in aller Welt sich unvorhersagbar verhält und damit auch dem formalen Kriterium des Konstruktionsbegriffs entspricht.

Eine weitere unvorhersagbare formale Eigenschaft ist die, dass in aller Welt nicht mit allen Interrogativformen verwendet werden kann. Ausgeschlossen ist welch-, sowohl als Determiner (20a) als auch als Pronomen (20b):

(20) a. *Welche Verbindung in aller Welt besteht zwischen Falco und Mozart?

b. A: Es gibt eine Verbindung zwischen Falco und Mozart.

$\mathrm{B}$ : *Und welche in aller Welt ist das?

Auch diese Beschränkung gilt nicht für alle emphatischen PPs (vgl. z.B. Num mußich nur noch mit dem richtigen Bein aufstehen. Welches, zum Teufel, war denn das? [L98]).

\footnotetext{
Mitte des 18. Jahthunderts Belege, in denen gar kein $W$ b-Ptonomen auftritt (i-ii), und auch Ende des 18. Jahrhunderts, als die Verwendung bereits auf $W$ b-Interrogative eingeschränkt war, tritt in aller Welt noch in klar appositiven Verwendungen (iii) und in kanonischen Adjunktpositionen (iv) auf:

(i) Ist es in aller Welt möglich? [G.E. Lessing, 1755]

(ii) Sagen Sie mir doch in aller Welt, Pater, ob Sie haben wollen, daß wir Christum verlassen, und seinem Widersacher dienen sollen? [F. Ambrosius, 1771]

(iii) Aber in aller Welt, was soll daraus werden und giebt es denn gar kein Mittel dagegen? [L. Tieck, 1796]

(iv) Wie hätt' ich denn in aller Welt darauf kommen sollen? [C.L. Heyne, 1786]
} 
Schließlich kann die PP in aller Welt ausschließlich mit Wh-Pronomen auftreten, die im Vorfeld eines Interrogativsatzes oder indirekten Fragesatzes stehen. Sie kann also nicht in Exklamativsätzen (21a), bei $\mathrm{Wh}$-Pronomen in Deklarativsätzen (21b) stehen, und auch nicht bei einem $W$-Pronomen in einem Interrogativsatz, wenn dieses nicht im Vorfeld steht $(22 \mathrm{~b}, \mathrm{c})$ :

(21) a. *Was in aller Welt für eine Naivität!

b. *Ich soll WAS in aller Welt tun?

a. Offensichtlich werden wieder Aktien gekauft. Aber wer kauft was? [A98]

b. ... Aber wer in aller Welt kauft was?

c. ...*Aber wer kauft was in aller Welt?

Es lässt sich argumentieren, dass (21a) aufgrund der semantischen Beschränkung in (7) ausgeschlossen ist, da Exklamativsätze keine offene Frage im Kopf des Sprechers voraussetzen. Bei (21b) greift ein solches Argument aber nur bedingt: Kontrastiv betonte $W h$-Pronomen in situ können zwar ebenfalls exklamativ gebraucht werden, der Satz ist aber auch dann inakzeptabel, wenn er tatsächlich als Frage gemeint ist. Bei (21b) handelt es sich um eine Echo-Frage, von der man möglicherweise sagen könnte, dass sie ebenfalls keine offene Frage im Kopf des Sprechers voraussetzt; bei (22c) handelt es sich aber eindeutig um eine echte Frage.

Auch in Relativsätzen kann WhIAW nicht auftreten (23a, b), selbst dann nicht, wenn inhaltlich identische indirekte Fragesätze völlig akzeptabel sind (vgl. 24a, b):

(23) a. *Die Regierenden dachten über das nach, was in aller Welt man tun könnte, um das Konsumklima im Land zu verbessern.

b. *Ich wüsste nichts, was in aller Welt Bands wie den Fleshtones „helfen“ könnte.

(24) a. ... wenn sich nun ... die Regierenden am Kopf kratzen und laut darüber nachdenken, was in aller Welt man wohl tun könne, um das Konsumklima im Land zu verbessern. [HAZ08]

b. Ich wüsste nicht, was in aller Welt Bands wie den Fleshtones ,helfen" könnte. [uww.flying-revolver.net]

Die Eigenschaften der WhIAW-Konstruktion können wie folgt zusammengefasst werden:

(25) WhIAW-Konstruktion

a. $\mathrm{S}_{\mathrm{i}}\left[\mathrm{L}_{\mathrm{NP}}\left[\mathrm{K} W\right.\right.$ h] ${ }_{\mathrm{PP}}$ in aller Welt $\left.] \mathrm{VP}\right]$

b. $S_{i}$ ist ein Hauptsatz oder

c. Argument eines Matrixprädikats $S_{i}$

d. $S_{i}$ ist eine offene Frage im Kopf von $\mathrm{X}$

e. X drückt Intensivierung und Unverständnis bezüglich $\mathrm{p}\left(\mathrm{S}_{\mathrm{i}}\right)$ aus 
f. X ist Sprecher von $\mathrm{S}_{\mathrm{i}}$ oder

g. Referent eines Arguments von $\mathrm{S}_{\mathrm{j}}$

Die Notation spielt dabei keine Rolle, entscheidend ist, dass in (25) das Wissen erfasst ist, das ein Sprecher haben muss, um WhIAW angemessen zu verwenden und um angemessene Verwendungen zu erkennen. ${ }^{14}$ Dieses Wissen beinhaltet Informationen bezüglich der syntaktischen (Oberflächen-)Struktur des Satzes, in dem das $W h$-Pronomen mit der intensivierenden PP steht, sowie ggf. über die Struktur des Matrixsatzes; es beinhaltet Informationen über die Bedeutung von WhIAW und über die Bedeutung des Satzes, in dem es auftritt, und es beinhaltet Informationen über den Sprecher und/oder die Referenten der Argumente des Matrixsatzes.

Aus grammatiktheoretischer Sicht ist nun die Frage entscheidend, wo ein syntaktisch-semantisch-pragmatischer Wissenskomplex wie der in (25) in einem Grammatikmodell verortet werden soll. Im Prinzip gibt es auf diese Frage vier mögliche Antworten: (a) in der Syntax, (b) im Lexikon, (c) im Weltwissen, oder (d) verteilt über Syntax, Lexikon und Weltwissen. Betrachten wir die Konsequenzen jeder dieser Möglichkeiten für ein Grammatikmodell:

(a) Wenn (25) in der Syntax verortet wird, lässt man damit semantisches und enzyklopädisches Wissen in der Syntax zu; die Syntax wird damit zu einem Konstruktikon im Sinne der Konstruktionsgrammatik. Ein Lexikon würde in diesem Fall überflüssig, da auch Wörter in einem solchen Konstruktikon repräsentiert werden können.

(b) Wenn (25) im Lexikon verortet wird, bedeutet dies, dass das Lexikon syntaktische Strukturen enthalten kann. Das Lexikon wird damit zu einem Konstruktikon im Sinne der Konstruktionsgrammatik. Eine Syntax würde in diesem Fall überflüssig, denn auch abstraktere syntaktische Strukturen könnten im Lexikon repräsentiert werden.

(c) Wenn (25) im Weltwissen verortet wird, bedeutet dies, dass das Weltwissen sowohl lexikalisches als auch syntaktisches Wissen beinhalten kann. Damit würden sowohl Syntax als auch Lexikon überflüssig; außerdem

14 Dabei spielt es keine Rolle, in welchem Format die Zusammenfassung der Eigenschaften von WhIAW vorgenommen wird. Es wäre z.B. vorstellbar, eine Transformationsregel für eine (optionale) ,,intensivierende W-Expansion“ zu formulieren, die, analog zu traditionellen phonologischen Regeln, die Bedingungen beschreibt, unter denen diese Expansion möglich ist: $[\mathrm{NP}[\mathrm{N} \mathrm{WH}]] \rightarrow\left[\mathrm{NP}[\mathrm{N} \mathrm{WH}][\mathrm{PP}\right.$ in aller Welt $] / \mathrm{S}_{\mathrm{i}}[\#-\mathrm{VP}]$

$$
\begin{aligned}
& \mathrm{S}_{\mathrm{i}} \text { ist ein Hauptsatz oder } \\
& \text { ein Argument eines Matrixsatzes } \mathrm{S}_{\mathrm{j}} \\
& \mathrm{S}_{\mathrm{i}} \text { ist eine offene Frage im Kopf von X } \\
& \mathrm{X} \text { drückt Unverständnis bezüglich } \mathrm{p}\left(\mathrm{S}_{\mathrm{i}}\right) \text { aus } \\
& \mathrm{X} \text { ist Sprecher von } \mathrm{S}_{\mathrm{i}} \text { oder } \\
& \text { Referent eines Arguments von } \mathrm{S}_{\mathrm{j}}
\end{aligned}
$$

Diese „Regel“ wäre aber inhaltlich identisch mit der Darstellung in (25) und sie würde unter den konstruktionsgrammatischen Konstruktionsbegriff fallen, da sie ein Form-Bedeutungspaar mit nicht-vorhersagbaren formalen und semantischen Eigenschaften beschreibt. 
bestünde keine kategorische Grenze mehr zwischen sprachlichem und nicht-sprachlichem Wissen (eine Vorstellung, die nicht notwendigerweise von allen Konstruktionsgrammatikern geteilt wird, die sich aber in der Ideengeschichte der Konstruktionsgrammatik wiederholt findet).

(d) Wenn (25) über Syntax, Lexikon und Weltwissen verteilt repräsentiert wird, müssen spezifische, nicht-vorhersagbare Verknüpfungen zwischen diesen Bereichen zugelassen werden. Damit entstünde wiederum ein Konstruktikon im Sinne der Konstruktionsgrammatik, das sich allenfalls durch seine modulare Struktur von der holistischeren Lösung in (c) unterscheide. Da diese modulare Struktur aufgrund der nicht-vorhersagbaren Verknüpfungen keine Autonomie der einzelnen ,Module“ zulassen würde, wäre dies aus konstruktionsgrammatischer Sicht wenig problematisch.

Unabhängig davon, welche Lösung man wählt, entsteht also ein Konstruktikon im Sinne der Konstruktionsgrammatik. Damit ist man nicht notwendigerweise gezwungen, die klassische Trennung von Syntax und Lexikon/ Weltwissen aufzugeben: Die Lösung in (b), die wohl am ehesten der Lösung entspricht, die in traditionellen generativen Modellen gewählt würde, erlaubt es natürlich theoretisch, eine unabhängige syntaktische Komponente beizubehalten, in der dann nur diejenigen syntaktischen Prozesse verortet werden, die völlig regelmäßig und vorhersagbar sind. Das logische Argument für die Existenz von Konstruktionen in der Grammatik bliebe davon unberührt, da es ja nur besagt, dass es Konstruktionen gibt, nicht, dass Konstruktionen die einzige Form sprachlichen Wissens darstellen. Allerdings entfällt die Notwendigkeit einer separaten syntaktischen Komponente. Eine solche Notwendigkeit lässt sich auch nicht ohne Weiteres aus der Idee einer Trennung zwischen Kerngrammatik und Peripherie ableiten, da die ,unregelmäßigen" Strukturen im Lexikon, wie die WhIAW-Konstruktion zeigt, in den Bereich der Kerngrammatik fallen können (zumindest dann, wenn man annimmt, dass Fragesätze in einer solchen Kerngrammatik zu verorten sind). Aus dieser Tatsache ergibt sich ein starkes Argument dafür, Konstruktionen als einzige Repräsentationsform grammatischen Wissens zu betrachten.

\section{Konstruktionen als einzige Repräsentationsform grammatischen Wissens}

\subsection{Das ökonomische Argument}

Ein konstruktionsverarbeitendes System, also ein Mechanismus, der es erlaubt Strukturen mit idiosynkratischen Eigenschaften zu verarbeiten, kommt auch mit regelhaften Strukturen (also mit solchen obne idiosynkratische Ei- 
genschaften) zurecht (vgl. z.B. Fillmore 1985; Kay 1997). Regelhafte Strukturen sind ja nichts grundsätzlich anderes als idiosynkratische Strukturen, sie sind nur weniger spezifiziert und damit in mehr Zusammenhängen verwendbar. Ein System, das beispielsweise die WhIAW-Konstruktion in (25) verarbeiten kann, kann auch die Struktur in (26) verarbeiten:

(26) Wh-Konstruktion ${ }^{15}$
a. $S_{\mathrm{i}}\left[\left[_{\mathrm{NP}}\left[_{\mathrm{N}} W\right.\right.\right.$ b $\left.] \mathrm{VP}\right]$
b. $S_{i}$ ist ein Hauptsatz oder
C.
Argument eines Matrixsatzes $S_{i}$

Die $W$-Konstruktion in (26) unterscheidet sich von der WhIAW-Konstruktion in (25) nur dadurch, dass letztere zusätzliche formale, semantische und pragmatische Informationen enthält.

Ein regelverarbeitendes System kann dagegen ausschließlich regelhafte Strukturen verarbeiten, da es keine Möglichkeit zur Verfügung stellt, Idiosynkrasien zu berücksichtigen. Natürlich kann man ein System postulieren, das einen $W h$-Satz wie Was hast du getan (vgl. 27a) nach allgemeinen Regeln aus (27b) ableitet:

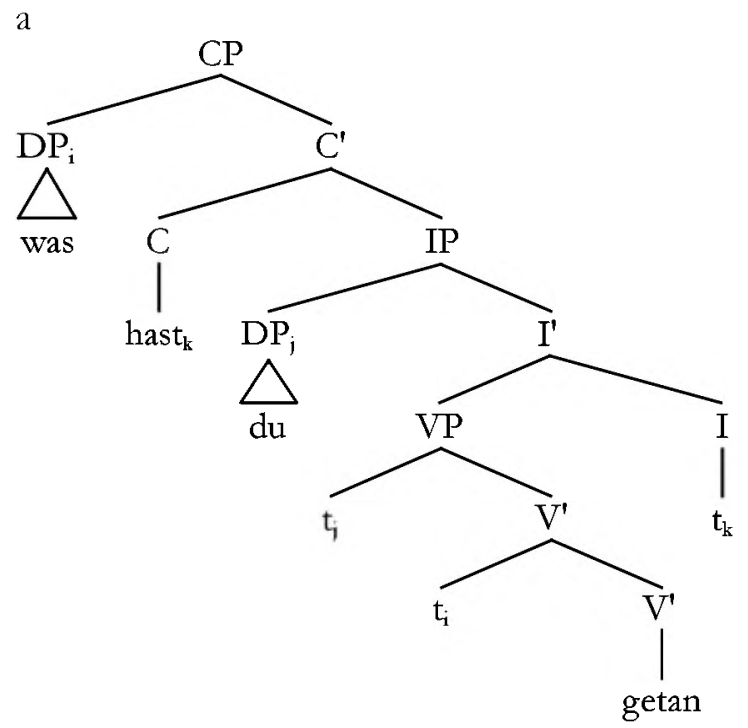

15 Natürlich soll (26) keine auch nur annähernd vollständige Darstellung der Eigenschaften von $W h$-Sätzen im Deutschen sein, sondern lediglich einen Versuch darstellen, die Gemeinsamkeiten aller $W b$-Sätze in einer Form zu erfassen, die (25) ähnelt. Dabei werden die Unterschiede zwischen Verbzweit- und Verbletztstellung ebenso ignoriert wie die Tatsache, dass das $W h$-Pronomen das Subjekt oder ein VP-internes Argument sein kann. Diese und weitere Unterschiede lassen sich aber grundsätzlich auf dieselbe Weise darstellen, z.B. $\mathrm{S}_{\mathrm{v} 2}\left[\mathrm{~L}_{\mathrm{NP}}\right.$

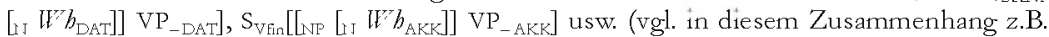
die HPSG-Analyse von deutschen Fragesätzen in Feldhaus 1999). 
b.

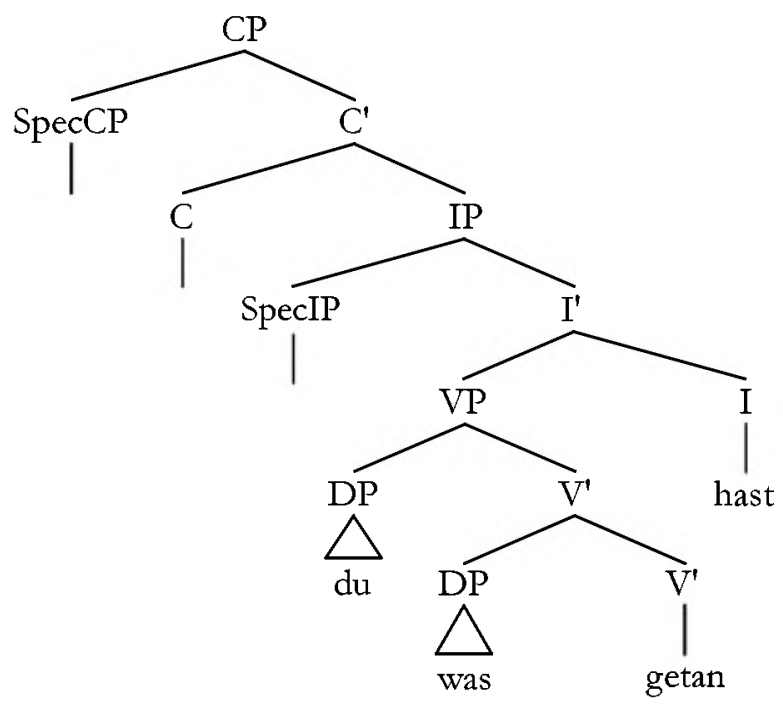

Allerdings kann dieses System den analogen Satz Was in aller Welt hast du getan nicht mit demselben Regelapparat erzeugen, da sich die formalen, semantischen und pragmatischen Beschränkungen nicht aus den in diesem Apparat vorhandenen Regeln ergeben. Es müssten also zusätzliche Regeln eingeführt werden, die eine Einbindung von semantischen und pragmatischen Informationen erlauben. Solche „Regeln“" wären aber von grundsätzlich anderer Art als die Regeln, die (27a) aus (27b) erzeugen und könnten nicht als Teil desselben Systems dargestellt werden; sie würden also ein separates System erfordern (z.B. ein mit den notwendigen Mechanismen versehenes Lexikon) $\cdot{ }^{16}$

Aus einem Modell, das von der Existenz eines regelverarbeitenden Systems ausgeht, ergibt sich deshalb notwendigerweise eine Grammatik mit (mindestens) zwei Systemen. Dagegen kommt ein Modell, das von vorneherein ein konstruktionsverarbeitendes System annimmt, mit einem einzigen System aus.

16 In der generativen Literatur werden einige der formalen Eigenschaften des englischen [WH the bell] damit erklärt, dass das emphatische the bellkein „D-Linking “ zulässt, also nicht auf einen in Diskurs erwähnten bzw. anderweitig identifizierbaren Referenten verweisen darf (Pesetsky 1987). Es ist jedoch nicht klar, inwiefern the bell tatsächlich kein D-Linking erlaubt oder inwiefern die syntaktischen Eigenschaften dieses Ausdrucks aus dieser Tatsache folgen (vgl. Szabolcsi/Zwarts 1993). Ob sich WhIAW und ähnliche deutsche Ausdrücke von WhPronomen im Allgemeinen überhaupt durch D-Linking unterscheiden, will ich nicht beurteilen (siehe dazu Wiltschko 1997); in jedem Fall scheint es nicht möglich, die spezifischen semantischen und syntaktischen Beschränkungen aus dieser Eigenschaft abzuleiten. 
Akzeptiert man also grundsätzlich die Existenz von Konstruktionen im sprachlichen Wissen, verlangt es das Sparsamkeitsprinzip, ein rein konstruktionsverarbeitendes System anzunehmen:

Jedes System, das nicht-kompositionelle und/oder syntaktisch nicht voll regelhafte Strukturen repräsentieren und zur Verarbeitung bereitstellen kann, kann auch kompositionelle und syntaktisch regelhafte Strukturen repräsentieren und zur Verarbeitung bereitstellen.

In den Köpfen von Sprechern existiert ein System, das nicht-kompositionelle und/oder syntaktisch nicht voll regelhafte Strukturen repräsentieren und zur Verarbeitung bereitstellen kann.

Es wäre unökonomisch, zusätzlich zu diesem ohnehin vorhandenen System ein zweites, regelverarbeitendes System anzunehmen.

Hier wird angenommen, dass eine Grammatik mit zwei Systemen (oder eine Grammatik, die um ein nicht-grammatisches System zur Verarbeitung von Konstruktionen ergänzt werden muss), weniger ökonomisch ist als eine Grammatik, die aus einem einzigen (nicht notwendigerweise sprachspezifischen) System besteht. Sparsamkeit wird also anhand der Anzahl benötigter Systeme gemessen. Dagegen ließe sich einwenden, dass eine Lösung mit zwei Systemen dann sparsamer sein könnte, wenn das eine System mit einer kleinen Zahl von Regeln den größten Teil der Grammatik beschreiben könnte, während das andere System nur einige wenige irreguläre Strukturen enthält. In diesem Fall wären die beiden Systeme gemeinsam möglicherweise deutlich kleiner als ein gemeinsames, das das gesamte sprachliche Wissen in Form von Konstruktionen erfasst. Bedenkt man aber, dass selbst das Lexikon im klassischen Sinne (d.h. als reiner Wortspeicher verstanden) für einen durchschnittlichen Sprecher selbst nach konservativen Schätzungen allein 17.000 Lexeme enthalten und zur sofortigen Verarbeitung bereitstellen muss (Goulden et al. 1990), so ist klar, dass Einsparungen von einigen Hundert Konstruktionen nicht ins Gewicht fallen würden, selbst wenn sie möglich sein sollten. ${ }^{17}$

Damit ist natürlich nicht bewiesen, dass die menschliche Sprachfähigkeit tatsächlich auf einem rein konstruktionsverarbeitenden System beruht, denn das Sparsamkeitsprinzip führt nicht notwendigerweise zum realistischsten Modell, aber es legt eine Forschungsstrategie nahe, die solange von einem rein konstruktionsverarbeitenden System ausgeht, bis dieses System an empirischer Evidenz scheitert.

$17 \quad$ Ein zwei-systemiges Modell wäre nicht nur deshalb unökonomisch, weil es gegen das wissenschaftstheoretische Sparsankeitsprinzip verstoßen würde und damit nur schwer zu rechtfertigen wäre, sondern auch, weil unklar wäre, wie ein solches System evolutionär entstehen sollte, da es zunächst nur eine ohnehin vorhandene Fähigkeit replizie ren und damit keinen Selektionsvorteil bringen würde. 


\subsection{Empirische Evidenz}

Die Frage nach der grundlegenden Architektur natürlichsprachlicher Grammatiken kann natürlich nicht auf der Grundlage des hier diskutierten logischökonomischen Arguments entschieden werden. Ökonomieargumente können leicht zu falschen Ergebnissen führen, sie müssen deshalb, wie jedes andere Argument, durch empirische Evidenz aus möglichst vielen Phänomenbereichen abgesichert werden.

Empirische Evidenz für ein konstruktionsverarbeitendes System als einziges (oder wenigstens dominantes) System natürlichsprachlicher Grammatiken findet sich aber in einer Reihe von Phänomenbereichen, vom Spracherwerb über die Untersuchung syntaktischer Irregularitäten auch in scheinbar regelgeleiteten Bereichen der Grammatik (die mindestens seit Lakoff 1970 und Ross 1967 einen festen Platz in syntaxtheoretischen Diskussionen haben sollte) bis hin zur Idiomforschung und Phraseologie.

Ich möchte hier nicht näher auf diese Bereiche eingehen, da sie die ausführliche Diskussion, die ihnen zusteht, an anderer Stelle erhalten. Die Forschung zum konstruktionsgestützten Spracherwerb wird ausführlich z.B. in Tomasello (2003) zusammengefasst; speziell zum Deutschen vgl. z.B. Behrens (2009, in diesem Band), vgl. auch die ausführliche Darstellung des Erwerbs von $W b$-Sätzen im Deutschen in Steinkrauss (2009). Die Erforschung syntaktischer Phänomene im Kernbereich der Grammatik, die sich nicht auf allgemeine Regeln reduzieren lassen, hat in Bezug auf das Deutsche gerade erst begonnen, siehe z.B. Jacobs (2008, 2009), Stefanowitsch (2006, 2009) und Webelhuth (in diesem Band). Als Einstieg in die neuere empirische Idiomforschung sei z.B. Wulff (2008) empfohlen, die u.a. zeigt, dass (wie in der Konstruktionsgrammatik erwartbar) ein fließender Übergang zwischen (scheinbar) idiomatischen und (scheinbar) kompositionellen Strukturen besteht.

Stattdessen möchte ich hier auf einen Phänomenbereich eingehen, der bisher wenig Beachtung gefunden hat: die systematische Nichtanwendung scheinbar allgemeiner „,Regeln“. In einem regelgestützten System dürfte es zwar eine gelegentliche unsystematische Nichtanwendung von Regeln (im Sinne von „Performanzfehlern ") geben, aber wenn Regeln systematisch nicht angewendet würden, wäre das ein klarer Hinweis auf holistisch repräsentierte Muster, bei deren Abruf und Kombination mit anderen Mustern und Konstruktionen keine allgemeinen Regeln zur Anwendung kommen (oder, um es konstruktionsgrammatisch auszudrücken: kein Abgleich mit allgemeineren, abstrakteren Konstruktionen stattfindet).

Ich werde das am Beispiel des Ausdrucks seinen/ihren Preis haben zeigen, der in (28a) schematisch in seiner häufigsten syntaktischen Form dargestellt ist (typische Beispiele sind 28b-c): 
(28) a. $\mathrm{NP}_{\mathrm{i}}$ hat POSS $_{1}$ Preis

b. Luxus hat seinen Preis: Ein Whirlpool von guter Qualität kostet rund 7000 bis 8000 Euro. [RH'Z06]

c. Doch Qualität hat ibren Preis, kostet viel Geld. [RHZ07]

d. NP hat POSS Ursache/n, Grund/Griunde, Stärken, Schwächen, Fehler, Tücken, Risiken, Probleme, Ordnung, Richtigkeit, Berechtigung, Gutes, Positives, Vorteile, Nachteile, Für und Wider, zwei Seiten, Grenze/n, Ausnahmen, Zeit, Stunde, Ende, ...

Der Ausdruck ist eine spezielle Variante eines abstrakteren Musters [NP. hat POSS $_{\mathrm{i}} \mathrm{NP}_{\mathrm{i}}$ ], für das in (28d) weitere typische Beispiele aufgeführt sind. Dieses abstraktere Muster scheint nicht voll produktiv zu sein; so sind z.B. die Ausdrücke sein/ihr Gutes/Positives/Schönes haben sehr konventionell, während sich die Antonyme sein/ibr Schlechtes/Negatives/Hässliches baben sehr selten bis nie finden. Dies ist keine allgemeine Beschränkung auf positiv konnotierte Wörter, da z.B. sowohl das Wort Vorteile als auch sein Antonym Nachteile häufig in dem Muster auftreten. Andererseits ist das Muster nicht auf die häufig darin verwendeten Substantive beschränkt, es kann problemlos auf ungewöhnlichere Substantive ausgedehnt werden:

(29) a. Aber alles hat seine Nachwirkungen, ich nehme das Zeug seit 12 Monaten, mir geht's soweit gut. [www.ht-mb.de]

b. $100 \mathrm{~V}$ oder $110 \mathrm{~V}$ ist dem Netzteil erfahrungsgemäß egal. Alles hat so seine Toleranzen. [www.embjapan.de]

c. Jedes System hat seine Bugs. [forum.ts.fujitsu.com]

Das Muster ist trotz seiner möglicherweise leicht eingeschränkten Produktivität semantisch weitgehend kompositionell: Es bedeutet so etwas wie „X hat eine Eigenschaft $\mathrm{Y}$, die für X typisch/erwartbar ist" ${ }^{c}$, wobei die Komponente „für X typisch/erwartbar" sich aus der Verwendung des Possessivpronomens ableiten lässt. Dafür spricht auch, dass sich ein analog konstruiertes Muster mit derselben Bedeutung auch in anderen Sprachen findet:

(30) a. For Americans, getting sick has its price [washingtonpost.com] „Für Amerikaner hat das Krankwerden seinen Preis."

b. Reste que virer Pellegrini a son prix: 4 millions d'euros selon les calculs du quotidien sportif espagnol AS. [sportune.fr] „Aber Pellegrini zu entlassen hat seinen Preis: 4 Millionen Euro, nach den Berechnungen der spanischen Sportzeitschrift AS. ${ }^{c}$

Das Muster ist außerdem syntaktisch regelhaft, es lässt sich z.B. mit allen regulär erwartbaren Wortstellungen und in allen Tempora verwenden, das Verb und das Possessivpronomen der Objekt-NP kongruieren mit dem Subjekt usw. 
In einer regelgestützten Grammatik gäbe es deshalb keinen Grund, das Muster in seiner Gesamtheit zu repräsentieren, es könnte bei Bedarf mit den vorhandenen Regeln aus den vorhandenen Lexikoneinträgen erzeugt werden. In der Konstruktionsgrammatik könnte das Muster aus allgemeineren Konstruktionen im Konstruktikon erzeugt werden, aufgrund des einheitlichen Repräsentationsformats im Konstruktikon könnte es bei entsprechender Gebrauchshäufigkeit aber auch einen eigenen Eintrag (oder mehrere Einträge für verschiedene häufige Varianten) erhalten.

Auf der Grundlage introspektiver Beispiele lassen sich diese beiden Möglichkeiten nicht unterscheiden, aber in Gebrauchsdaten wäre, wie oben angedeutet, ein Unterschied zu erwarten: Wenn das Muster für jedes Gebrauchsereignis aus allgemeinen Regeln erzeugt würde, müssen diese Regeln (von vereinzelten unsystematischen Versprechern abgesehen) vollständig zur Anwendung kommen. Wenn das Muster dagegen holistisch repräsentiert wird und nur diese holistische Repräsentation abgerufen wird, könnten dabei einzelne allgemeinere Regeln systematisch verletzt werden, da sie in einem konkreten Gebrauchsereignis nicht zur Anwendung kommen müssten.

Genau das ist bei dem Muster in (28a) der Fall: Im Deutschen Referenzkorpus findet sich eine beträchtliche Zahl von Beispielen wie den folgenden, bei denen das Possessivpronomen der Objekt-NP nicht mit dem femininen Subjekt kongruiert, sondern stattdessen in der maskulinen/neutralen Form steht:

(31) a. Das aber bedeutet: Qualität hat seinen Preis - bei MTB's kann der in Regionen von 5000 Mark und mehr gehen. [M95]

b. ... denn die anwaltliche Vertretung vor zwei Instanzen hat seinen Preis. [A99]

c. Aber Boppard ist nicht irgendeine Kleinstadt, sondern Tourismusmetropole und Mittelzentrum. Diese Doppelfunktion hat seinen Preis. [RHZ99]

d. Doch die harte Selektion auf dem Weg zum bayerischen Gymnasium hat seinen Preis ... [NUZ05]

Wenn diese Sätze, wie durch ein regelgestütztes Grammatikmodell vorhergesagt, unsystematische Versprecher (,Performanzfehler ${ }^{c}$ ) wären, würden wir erwarten, dass auch der umgekehrte Fall von maskulinen/neutralen Subjekt-NPs mit femininem Possessivpronomen in der Objekt-NP existiert. Und tatsächlich finden sich derartige Beispiele:

a. Der Zugang zur letzten Meile hat ihren Preis [NUZ06]

b. Der Einstieg ins ernsthafte journalistische Geschäft bei der ARD hat ihren Preis. [NUZ06] 
c. Schon nach nur wenigen Stunden Unterricht kann Billie mit auswendig gelernten Floskeln wie ,Eine Supermacht qu sein, hat ihren Preis" etwa die brisante Frage nach der Notwendigkeit der Anwesenheit der US-Soldaten im Nahen Osten beantworten. [RHZ05]

Bei der Verteilung der inkongruenten Possessivpronomen zeigt sich aber ein drastisches Missverhältnis zwischen maskulinen/neutralen und femininen Possessivpronomen. Von insgesamt 1.085 Beispielen dieses Musters mit maskulinen oder neutralen Subjekt-NPs finden sich nur drei, in denen das Possessivpronomen feminin ist. Bei den insgesamt 1.129 Beispielen mit femininen Subjekt-NPs finden sich dagegen 40, in denen das Possessivpronomen maskulin/neutral ist (vgl. Tabelle 1, in der die statistisch erwarteten Zufallswerte in Klammern angegeben sind). Dieser Unterschied ist höchst signifikant (Fisher-Yates, $\mathrm{p}<0.001) .{ }^{18}$

\begin{tabular}{|l|c|c|c|}
\hline & Mask./Neut. & Fem. & Gesamt \\
\hline Kongruent & $\begin{array}{c}1082 \\
(1064)\end{array}$ & $\begin{array}{c}1089 \\
(1107)\end{array}$ & 2171 \\
\hline Inkongruent & 3 & 40 & 43 \\
& $(21)$ & $(22)$ & \\
\hline Gesamt & 1085 & 1129 & 2214 \\
\hline
\end{tabular}

Tab. 1: Häufigkeit von kongruenten und nicht-kongruenten Possessivpronomen im Muster $\left[\mathrm{NP}_{\mathrm{i}}\right.$ hat POSS ${ }_{\mathrm{i}}$ Preis] (Deutsches Referenzkorpus, Archiv W)

In einem konstruktionsgestïtzten Grammatikmodell erklärt sich diese Asymmetrie auf natürliche Weise, wenn man annimmt, dass im Konstruktikon die Muster mit dem maskulinen/neutralen bzw. dem femininen Possessivpronomen jeweils eigene Repräsentationen haben (d.h., dass es getrennte (aber natürlich verknüpfte) Einträge für $\left[\mathrm{NP}_{\mathrm{i}}\right.$ bat seinen Preis $]$ und $\left[\mathrm{NP}_{\mathrm{i}}\right.$ bat ibren Preis $]$ gibt), und dass die Variante mit dem maskulinen/neutralen Pronomen kognitiv stärker verankert wäre. Von diesen Repräsentationen würde dann normalerweise diejenige ausgewählt, die zum Genus der in die Leerstelle eingefügten Subjekt-NP passt. Da aber keine allgemeine Kongruenzregel zur Anwendung käme, würde die $\left[\mathrm{NP}_{\mathrm{i}}\right.$ bat seinen Preis $]$ aufgrund ihrer stärkeren Verankerung auch dann manchmal gewählt werden, wenn die SubjektNP feminin ist.

18 Tatsächlich ist die Asymmetrie zwischen den Kongruenzfehlern mit maskulinen und denen mit femininen Possessivpronomen noch größer als der statistische Unterschied suggeriert. Die Beispiele für letztere, die in (32) komplett aufgelistet sind, lassen sich in zwei Fällen klar als Flüchtigkeitsfehler analysieren, bei denen eine feminine NP (Meile, $A R D$ ) zwischen dem Subjekt und dem Possessivpronomen steht und zur femininen Kongruenz verleitet; der dritte Fall ist sogar ein direktes nicht-muttersprachliches Zitat. Bei den maskulinen Kongruenzfehlern gibt es manchmal eine entsprechende maskuline NP zwischen Subjekt und Possessivpronomen (Gymnasium in [31d]), aber in den meisten Fällen ist dies dort nicht der Fall. 
Diese Erklärung kann natürlich nur dann überzeugen, wenn es einen unabhängigen Grund für die Annahme gibt, dass die maskuline/neutrale Variante des Musters stärker verankert ist. Ein solcher Grund könnte entweder in der jeweiligen Häufigkeit der beiden Varianten oder in der Häufigkeitsverteilung von Wörtern innerhalb jeder der Varianten liegen.

Die Häufigkeit der beiden Varianten unterscheidet sich nicht sehr stark: Die Variante mit dem maskulinen/neutralen Pronomen macht 51 Prozent aus, die mit dem femininen 49 Prozent. Ein solcher Unterschied dürfte kaum reichen, um das eine Muster sehr viel stärker zu verankern als das andere. Und tatsächlich ist ohnehin nicht anzunehmen, dass die reine Häufigkeit einer Struktur die hier beobachtete Art von Kongruenzfehlern beeinträchtigen könnte, da in diesem Fall zu erwarten wäre, dass solche Fehler überall dort auftreten müssten, wo eine Variante eines sprachlichen Ausdrucks häufiger als die anderen Varianten ist.

Anders sieht es bei der Verteilung der Subjekt-NPs innerhalb der beiden Varianten aus: Diese zeigt eine Asymmetrie, die potenziell eine Erklärung für die beobachteten Kongruenzfehler liefern könnte. Tabelle 2 zeigt die jeweils zehn häufigsten Substantive in der Subjektposition der beiden Muster.

\begin{tabular}{|c|c|c|c|}
\hline \multicolumn{2}{|c|}{$\left[\mathrm{NP}_{i}\right.$ hat seinen Preis $]$} & \multicolumn{2}{|c|}{$\left[\mathrm{NP}_{i}\right.$ bat ibren Preis $]$} \\
\hline Subjekt-NP & Häufigkeit & Subjekt-NP & Häufigkeit \\
\hline das & 213 & Qualität & 201 \\
\hline alles & 121 & Sicberbeit & 48 \\
\hline Erfolg & 31 & Schönheit & 30 \\
\hline das alles & 25 & Freibeit & 27 \\
\hline Luxus & 25 & Arbeit & 23 \\
\hline Qualität & 14 & Technik & 22 \\
\hline der & 14 & die & 18 \\
\hline Aufwand & 13 & Exklusivität & 18 \\
\hline Angebot & 12 & Demokratie & 15 \\
\hline Fortschritt & 11 & Leistung & 15 \\
\hline
\end{tabular}

Tab. 2: Die zehn häufigsten Subjekte im Muster [ $\mathrm{NP}_{\mathrm{i}}$ bat seinen/ibren Preis] (DeReKo, Archiv W)

Bei der Variante mit maskulinem/neutralem Possessivpronomen machen die beiden häufigsten Muster das hat seinen Preis und alles hat seinen Preis (unter Einbeziehung des kombinierten das alles hat seinen Preis) fast ein Drit-

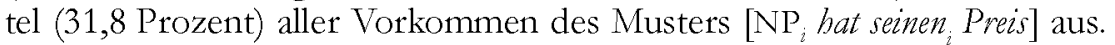
Zwischen diesen beiden Mustern und dem nächsthäufigsten Ausdruck Erfolg hat seinen Preis fällt die Häufigkeit auf ein Zehntel ab. 
Bei der Variante mit femininem Possessivpronomen gibt es dagegen keinen Ausdruck, der sich in seiner Häufigkeit derartig stark absetzt. Der häufigste Ausdruck Qualität hat ihren Preis macht zwar immerhin ein Fünftel (18,5 Prozent) des Musters $\left[\mathrm{NP}_{i}\right.$, hat ibren Preis] aus, er ist damit aber nur viermal häufiger als die nächsthäufigste Subjekt-NP Sicherheit.

Angesichts dieser klaren Häufigkeitsunterschiede ist es durchaus plausibel anzunehmen, dass die Variante [(das/alles) bat seinen Preis] aufgrund ihrer relativen Häufigkeit ein stark verankertes eigenständiges Muster darstellt und so dazu beiträgt, dass auch das abstraktere Muster [NP hat seinen $_{\mathrm{i}}$ Preis $]$ insgesamt stärker verankert ist als die Variante mit dem femininen Possessivpronomen. Auf dieses stärker verankerte Muster greifen Sprecher/innen dann häufig auch in den Fällen zu, in denen sie eine feminine Subjekt-NP in die Leerstelle einsetzen. Die Kongruenz von Subjekt-NP und Possessivpronomen wird dabei nicht überprüft. Solche systematischen Regelverletzungen im Kontext spezifischer grammatischer Muster stellen klare empirische Evidenz für ein konstruktionsgestütztes Grammatikmodell dar, vor allem, wenn sich weitere ähnliche Fälle finden; ob dies der Fall ist, bleibt abzuwarten.

\section{Schlussbemerkungen}

Die Grammatik natürlicher Sprachen wird in der Sprachwissenschaft ganz selbstverständlich als regelverarbeitendes System betrachtet. Diese Sichtweise hat eine lange Tradition, deren Ursprünge weit vor der Entstehung generativer Grammatiktheorien liegen; vor dem Aufkommen beschränkungsgestützter Modelle ist sie nie ernsthaft infrage gestellt worden (und auch in Letzteren liegt das Hauptaugenmerk auf der Modellierung von Regularitäten). Die Selbstverständlichkeit dieser Tradition führt dazu, dass schon bei der Suche nach theorierelevanter Evidenz, und erst recht bei deren Interpretation, Phänomene bevorzugt werden, die sich in eine regelgestützte Sichtweise einfügen.

Angesichts dieser Tradition ist nicht zu erwarten, dass sich eine alternative Sichtweise schnell durchsetzen wird, selbst wenn sie, wie die Konstruktionsgrammatik, durch eine wachsende Zahl methodisch ausgereifter empirischer Studien aus dem Spracherwerb, der Psycholinguistik, der diachronen Linguistik, der Typologie und zunehmend auch der Syntaxtheorie gestützt wird.

Die Diskussion um die Architektur menschlicher Sprachen wäre aber wissenschaftlich sauberer, wenn das logisch-ökonomische Argument als syntaxtheoretische Nullhypothese etabliert würde. Anstatt davon auszugehen, dass Sprache regelgestützt ist, und alles, was diesen Regeln nicht gehorcht, ins Lexikon abzuschieben, sollte die Sprachwissenschaft mit der einfacheren 
Hypothese anfangen, dass die kognitiven Prozesse, die uns die Verarbeitung von idiosynkratischen Konstruktionen auf den verschiedensten Komplexitäts- und Abstraktionsstufen ermöglichen, auch zur sehr viel weniger anspruchsvollen Verarbeitung regelhafter Strukturen befähigen.

Die beiden hier vorgestellten Fallstudien zeigen, wie theoretisch motivierte empirische Analysen einzelner Muster und Konstruktionen dazu beitragen können, die Reichweite eines konstruktionsgestützten Grammatikmodells zu erforschen. Die erste Studie zeigt, wie unvorhersagbare formale und semantische Eigenschaften einzelner Konstruktionen direkt in die Kerngrammatik hinein wirken und deshalb nicht als randständige Ausnahmefälle betrachtet werden können. Die zweite Studie zeigt anhand systematischer Regelverletzungen, dass auch vollständig (oder weitgehend) vorhersagbare Syntagmen in ihrer Gesamtheit repräsentiert und verarbeitet werden können. Diese Art von Regelverletzung stellt möglicherweise die beste Evidenz für ein konstruktionsgestütztes Grammatikmodell dar, die sich außerhalb der Spracherwerbsforschung und der Psycholinguistik finden lässt.

Fallstudien einzelner Konstruktionen sind natürlich potenziell immer dem Vorwurf einer geringen Reichweite ausgesetzt. Sie sind aber für die konstruktionsgrammatische Forschung in der aktuellen Phase der Theoriefindung und Theoriediskussion unvermeidlich; sieht man von der HPSG ab, so bieten Konstruktionsgrammatiken derzeit kein Grammatikmodell, das zur vollständigen Beschreibung einer bestimmten Sprache taugt. Konstruktionsgrammatiken sollen auch eher Theorien darüber sein, wie sprachliches Wissen erlernt, repräsentiert und in der Sprachproduktion angewendet wird. Bei allen unüberbrückbaren theoretischen Differenzen zur traditionellen Generativen Grammatik, wie sie im vorliegenden Band etwa von Gereon Müller vertreten wird, ist dies interessanterweise eine Eigenschaft, die die beiden theoretischen Lager eint: Auch die traditionelle Generative Grammatik hat nie eine auch nur annähernd vollständige Beschreibung einer Sprache hervorgebracht - unser Wissen über Sprache und notwendige sprachwissenschaftliche Fragestellungen hat sie trotzdem, und trotz ihrer deskriptiven Grenzen, ein großes Stïck vorangebracht.

\section{Daten}

Deutsches Referenzkorpus (DeReKo) am Institut für Deutsche Sprache, Mannheim, Archiv der geschriebenen Sprache (W-öffentlich). Internet: www.ids-mannheim.de/ kl/projekte/korpora (Stand: 20.12.2010). 


\section{Literatur}

Abeillé, Anne/Schabes, Yves (1989): Parsing idioms in lexicalized TAGs. In: Proceedings of the Fourth Conference on European Chapter of the Association For Computational Linguistics. Morristown, NJ, S. 1-9.

Behrens, Heike (2009): Konstruktionen im Spracherwerb. In: Zeitschrift für Germanistische Linguistik 37, S. 427-444.

Behrens, Heike (in diesem Band): Die Grenzen des lexikalischen Lernens: Konstruktionsprozesse im Spracherwerb.

Diessel, Holger (1997): Verb-first constructions in German. In: Verspoor, Marjolijn/ Dong, Lee Kee/Sweetser, Eve (Hg.): Lexical and syntactical constructions and the construction of meaning: proceedings of the bi-annual ICLA meeting in Albuquerque, July 1995. Amsterdam u.a., S. 51-68.

Feldhaus, Anke (1999): German Wh-interrogatives in HPSG. In: Kordoni, Valia ( $\mathrm{Hg}$ ): Tübingen studies in head-driven phrase structure grammar. (= Arbeitspapiere des SFB 340, 132). Tübingen, S. 459-493.

Fillmore, Charles J. (1985): Syntactic intrusions and the notion of grammatical construction. In: Proceedings of the Eleventh Annual Meeting of the Berkeley Linguistic Society, S. 73-86.

Fischer, Kerstin/Stefanowitsch, Anatol (2006): Konstruktionsgrammatik: Ein Überblick. In: Fischer/Stefanowitsch (Hg.), S. 3-17.

Fischer, Kerstin/Stefanowitsch, Anatol (Hg) (2006): Konstruktionsgrammatik. Von der Anwendung zur Theorie. (= Stauffenburg Linguistik 40). Tübingen.

Goldberg, Adele E. (1995): Constructions: a Construction Grammar approach to argument structure. Chicago.

Goldberg, Adele E. (2006): Constructions at work. The nature of generalization in language. Oxford u.a.

Goulden, Robin/Nation, Paul/Read, John (1990): How large can a receptive vocabulary be? In: Applied Linguistics 11, S. 341-363.

Grimm, Jacob/Grimm, Wilhelm (1854-1960): Deutsches Wörterbuch. 16 Bde. Leipzig.

Holler, Anke (2007): Interrogativum. In: Hoffmann, Ludger (Hg.): Handbuch der deutschen Wortarten. Berlin/New York, S. 445-482.

Jacobs, Joachim (2008): Wozu Konstruktionen? In: Linguistische Berichte 213, S. 3-44.

Jacobs, Joachim (2009): Valenzbindung oder Konstruktionsbindung? Eine Grundfrage der Grammatiktheorie. In: Zeitschrift für Germanistische Linguistik 37, S. 490513.

Kay, Paul (1997): Construction grammar. In: Kay, Paul (Hg): Words and the grammar of context. (= CSLI Lecture Notes 40). Stanford, S. 123-131.

Kay, Paul/Fillmore, Charles (1999): Grammatical constructions and linguistic generalizations: the What's X doing Y construction. In: Language 75, S. 1-34. 
Lakoff, George (1970). Irregularity in syntax. New York u.a.

Lakoff, George (1987): Women, fire, and dangerous things: what categories reveal about the mind. Chicago u.a.

Langacker, Ronald W. (1987): Foundations of Cognitive Grammar: theoretical prerequisites. Stanford.

Leiss, Elisabeth (2009): Sprachphilosophie. Eine Einführung in die Axiomatik. Berlin.

Müller, Gereon (in diesem Band): Regeln oder Konstruktionen? Von verblosen Direktiven zur sequenziellen Nominalreduplikation.

Müller, Stefan (2006): Resultativkonstruktionen, Partikelverben und syntaktische vs. lexikonbasierte Konstruktionen. In: Fischer/Stefanowitsch (Hg.), S. 177-202.

Pesetsky, David (1987): Wh-in-situ: movement and unselective binding. In: Reuland, Eric J./ter Meulen, Alice G. B. (Hg.): The representation of (in)definiteness. (= Current Studies in Linguistics Series 14). Cambridge, MA u.a., S. 98-129.

Ross, John R. (1967): Constraints on variables in syntax. Diss. Cambridge, MA. [Neu aufgelegt als Ross, John R. (1986): Infinite syntax! Norwood, NJ.]

Sailer, Manfred (2003): Combinatorial semantics and idiomatic expressions in headdriven phrase structure grammar. (= Arbeitspapiere des SFB 340,161). Stuttgart/ Tübingen.

Stefanowitsch, Anatol (2006): Konstruktionsgrammatik und Korpuslinguistik. In: Fischer/Stefanowitsch (Hg.), S. 151-176.

Stefanowitsch, Anatol (2009): Bedeutung und Gebrauch in der Konstruktionsgrammatik: Wie kompositionell sind modale Infinitive im Deutschen? In: Zeitschrift für Germanistische Linguistik 37, S. 565-592.

Steinkrauss, Rasmus (2009): Frequency and function in WH-question acquisition. A usage-based case study of German L1 acquisition. Diss. Groningen.

Szabolcsi, Anna/Zwarts, Frans (1993): Weak islands and an algebraic semantics of scope taking. In: Natural Language Semantics 1, S. 235-284.

Tomasello, Michael (2003): Constructing a language: a usage-based theory of language acquisition. Cambridge, MA u.a.

Wahrig-Burfeind, Renate (Hg.) (2006): Wahrig Deutsches Wörterbuch. 8., vollst. neu bearb. u. aktual. Aufl. Gütersloh.

Webelhuth, Gerd (in diesem Band): Paradigmenwechsel rückwärts: die Renaissance der grammatischen Konstruktion.

Wiltschko, Martina (1997): D-linking, scrambling, and superiority in German. In: Groninger Arbeiten zur Germanistischen Linguistik 41, S. 107-142.

Wulff, Stefanie (2008): Rethinking idiomaticity: a usage-based approach. London/New York. 